$$
\text { "tmcs-kantor-fazekas" — 2011/5/15 — 16:11 — page } 137 \text { — \#1 }
$$

\title{
Conventions of mathematical problems and their solutions in Hungarian secondary school leaving exams
}

\author{
SÁndor KÁntor and Anna K. FAZEKAS
}

\begin{abstract}
Collecting and analyzing the conventions indispensable for interpreting mathematical problems and their solutions correctly assist successful education and objective evaluation. Many professional and didactic questions arose while collecting and analyzing these conventions, which needed clarification, therefore the materials involved concisely in the conventions enrich both the theory and practice of mathematics teaching. In our research we concentrated mainly on the problems and solutions of the Hungarian school leaving examinations at secondary level in mathematics.
\end{abstract}

Key words and phrases: mathematics teaching, conventions, standardised problem posing.

ZDM Subject Classification: Q30, C50.

\section{Introduction}

It is widely accepted that all problem-posers and problem-solver use conventions, since it is impossible to write down every detail of a problem. Conventions make it possible for professional register to be interpreted correctly, therefore they might be considered a part of the academic genre. These conventions evolve naturally in face-to-face interactions between teachers and their students, so there is no need to define them.

These conventions are also very important in the wider academic society where teachers and students work together, e.g. in the case of the school leaving

Copyright (C) 2011 by University of Debrecen 


$$
\text { "tmcs-kantor-fazekas" — 2011/5/15 — 16:11 — page } 138 \text { — \#2 }
$$

examinations or national competitions. In these cases, however, the conventions of the genre will not evolve automatically between the users, so their evolution needs to be consciously assisted. It is not easy to find standardised conventions and have them accepted, although they would be necessary, since details are of vital importance in many cases where the stakes are high.

We are dealing with the conventions of problems and official solutions of the Hungarian secondary school final written exams in mathematics, because these are easily accessible, detailed and standardised. Mostly the tests of the twolevel school-leaving exams of the past six years have been analysed, but we also included some problems of earlier school-leaving exams in our analysis. This review of the Hungarian material might be useful for international competitions and tests organised beyond our borders as well. It would be a good idea to investigate curricula and course books, too, but the curricula are not detailed enough, and the course books are not standardized. The conventions reported here are without complexity. We mainly deal with solving equations which is sometimes very problematic and with spectacular geometry from the former topics and, from the new topics we chose the topics of functions and arithmetic. We are going to illustrate with the use of examples that it is the topic of probability that needs collecting conventions and having them accepted most. This work has already started, but its results will be reported later.

It is important to note that the material reported here does not only reflect our ideas, but we also asked and used the opinion and ideas of several experts teaching either at secondary schools or colleges.

We have divided the conventions reported in this article into two main groups: the first group consists of conventions totally or partially (after some suggested refinements totally) agreed with by all. The second group includes controversial conventions which need certain modifications, but would be required by all means. The conventions in both groups were put into three subgroups according to their type: general conventions, conventions of specific topics and legal conventions. The latter name might seem unusual, but it will be revealed that they refer to specific mathematical content.

\section{Group I. Valid conventions}

\subsection{General conventions}

- G1. All statements must be proved while solving a problem.
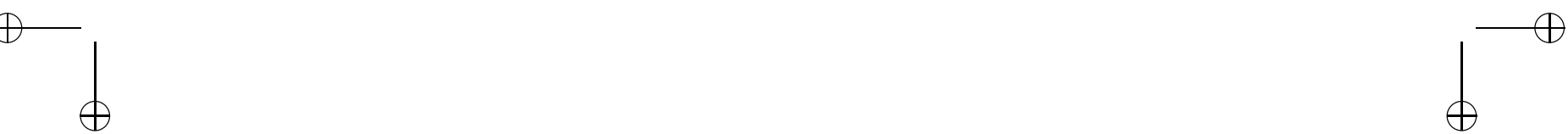
"tmcs-kantor-fazekas" — 2011/5/15 — 16:11 — page 139 — \#3

REMARK 1. This convention must be emphasized because the text of a problem often requires different actions, such as 'how many', 'where is', or 'find', etc. All statements written down must still be proved.

REMARK 2. It is an exception to this convention, of course, when the form of the problem (any multiple choice tests) or the exact words of the text (includes that proof is not required) make it evident.

Belonging to the above convention there are some conventions, independent of any specific topics, pertaining to specific proofs.

- G1.1. Trial of all possible events counts a thoroughly effective proof.

- G1.2. A design made to solve a problem does not prove anything. Remark. In geometry (especially in solid geometry, e.g. when making cross sections) reference to graphical approach is often acceptable, but reference to the design is not.

- G1.3. Reference to symmetry (e.g. in equation systems or geometric designs) must be detailed.

- G1.4. Proof carried out on an arbitrary element of a set is valid for all the elements of the set, but it must be indicated.

- G1.5. Trivial proofs (transformations, calculations) easily done mentally need not be written down.

- G1.6. Theorems known in higher mathematics but not part of the secondary curriculum may be referred to without proof or reference to their source. (The theorem must certainly be written down.)

- G2. The use of singular or plural nouns in the text of a problem requesting certain data does not give any information regarding the number of data.

REMARK 1. It is a very important convention, shared by all teachers, the awareness of which must be raised in students as well. For instance, the text "find the point" is not incorrect even if there are several points or there is not any. In such cases the candidate must find all of them, or prove that there is no such point.

REMARK 2. An exception is the case when the text requires one datum, but it is well-known that there is an infinite number of data. Let us show an example of this. The problem requires the specific, unequivocally defined 


$$
\text { "tmcs-kantor-fazekas" — 2011/5/15 — 16:11 — page } 140 \text { — \#4 }
$$

equation of a line. Here the problem-solver can choose to give one from the infinite number of equations of the specific line.

- G3. Approximate values should in a way be distinguished from actual values (e.g. with a swung dash).

REMARK. The basic concepts, methods and possibilities of calculations with the use of a calculator must be known to everyone, otherwise there is no point in assigning arithmetical tasks. It is against the goal of educating students to think that nowadays you encounter more and more problems, in which neither its poser nor its solver knows (or indicates) what is actually being counted. For example, when performing several operations on the same rounded off value, generally the solution given is not the rounded off value of the quantity to be found (which is originally required by the text), but something else, in a more favourable case it is the approximate value, without the rate of approximation indicated.

- G4. Notations used in the solution of a problem must be those already used in the text of it, or else they must be defined in the solution. In geometrical problems notations might be defined with designs but it must be indicated that you are using the notations of the design.

REMARK. Official solutions always corresponds to $\mathbf{G 4}$, so we include it here in default of unambiguous agreement.

- G5. The question raised in the problem must always be answered.

REMARK. The answer cannot be accepted if the exact answer to the question posed in the problem can only be reached by deductive reasoning.

- G6. If the problem has got several parts formally, then the conditions must be declared in each part to be valid everywhere (they are not automatically valid in the different parts).

REMARK. For example, when the text of a problem divides into part a and $b$ after an introductory part, the conditions included in the introduction are valid for both parts, but the conditions included in part a must be repeated in part $b$ in case they are intended to be valid for that part as well. 


$$
\text { "tmcs-kantor-fazekas" — 2011/5/15 — 16:11 — page } 141 \text { — \#5 }
$$

\subsection{Conventions of specific topics}

- S1. The text "solve the following equation at the domain of Real Numbers" means finding all the real values for the variables that will give a true statement when substituted for the variable, including multiple roots.

Remark. As a result of convention $\mathbf{G 1}$ the roots must be proved to be the solutions of the equation. However, the domain need not be given in the solution in case it was not used to find the roots. If the poser of the problem considers giving the domain part of the solution, then they need to indicate this requirement in the text of the problem.

- S2. If the equations written under one another are not considered consequence equations, their nature must be indicated.

REMARK 1. Remarks written between equations written under each other (e. g. "combining like terms", "squared") do not define the relation the candidate had in mind, therefore they do not absolve S2.

REMARK 2. We insist on recording a convention on this question even if there is no total agreement. The version recorded here matches most with practice and it is detailed in [1] that this one is the easiest to handle.

- S3. In the case of equations gained from the text of a problem the fact that the equation is not considered the consequence of the text must be indicated as in the case of equations written under each other.

- S4. Addition of equations or inequalities means addition of the expressions of the corresponding sides.

- S5. A quadratic equation with real coefficients cannot have only one real root. There may be either no real roots or two real roots which might sometimes be identical (a double root).

Remark. For higher degree equations the convention is a reasonable generalization of the above one.

- S6. When the text of a problem says "Construct...", the steps of the construction must be detailed and the method must be proved. The construction itself need not be carried out. 


$$
\text { "tmcs-kantor-fazekas" — 2011/5/15 — 16:11 — page } 142 \text { — \#6 }
$$

REMARK. Many people think that the conditions of solubility and their proof also belong to the solution of construction problems. According to our experience the difficult proof of the discussion is not considered to be part of the solution, therefore there is no proposition here in this question.

- S7. In geometric designs the order of nominating letters indicate a circle cycle.

- S8. You can only require the curve of a function which is defined at the domain of a limited set.

REMARK. Since it is only possible to draw the curve of such a function, there is no other possibility for the problem to be solvable. For example the function defined by the formulae $f(x)=x$ at the domain of the real numbers, cannot be drawn, only if it is confined to a finite set.

- S9. Sets (e.g. the solution set of an equation) must be well-defined and detailed.

REMARK. When defining the period of a trigonometric function for instance, reference to the fact that $k$ is usually a whole number is not enough, it must be indicated.

\subsection{Legal conventions}

- L1. Students have the right to know - on the basis of the text of the problem and previously learnt, curriculum-based conventions - what their tasks are while solving a problem (how they can score maximum).

- L2. Students have the right to know what principles are used while correcting their tests. E.g. what the consequences of their miscounts are.

- L3. If the literal interpretation of a problem is unambiguous the solution according to any interpretation must be fully acceptable.

REMARK. We hope that there will be no need for such a convention in the future. 
3. Group II. Controversial conventions, but by all means necessary in a certain form

\subsection{General conventions}

- CG1. As many consecutive statements must be included in the proof as needed for a good student to understand the deductive steps clearly.

REMARK. The question of how detailed a proof must be has always been a controversial one. Educational practice is subjective. Almost everyone has a different idea and it is impossible to reach an agreement on this topic. Most teachers suggest their students that they should give a detailed proof in their solutions, but most students do not follow this advice.

According to the official solutions of the final examinations a proof which is less detailed than the official one might be accepted as well. It is a very broad definition, so it is useless. We suggest students to make references pertaining to how they see the possibility of the proof and how easily they could provide it. Such references are for instance the expressions "obviously", "naturally", "abstract nonsense", which are called text for reserving rights, since you can refer to them when complaining about the results after having obtained poor scores because of the proof.

- CG2. Discussion of degenerate cases may be skipped over when they are obvious in the steps of the proof.

Remark. Herein we mean the degenerate cases evolving through the proof, not the degenerate cases of the theorem.

- CG3. If the text of the problem contain units, the solution must contain units of the same type.

\subsection{Conventions of specific topics}

- CS1. When solving an equation is only a means of solving a problem it need not be detailed as much as when the task is the solution of the equation itself.

- CS2. Neither the variables, nor the numbers in equations derived from the text of a problem can take units. They can only include mathematical objects (numbers, vectors, functions, etc.) 


$$
\text { "tmcs-kantor-fazekas" — 2011/5/15 — 16:11 — page } 144 \text { — \#8 }
$$

REMARK. The misuse of units is a double mistake: a conceptual one and a pragmatic one!

- CS3. When requiring the plot of a graph of a function where the graph does not consist of straight lines, you must give all the abscissas which are needed to plot the graph.

REMARK 1. Otherwise readings from the graph might be incorrect, although the students made no mistakes while plotting it, since e.g. monotony or convexity are valid.

REMARK 2. It is not only plotting the graph of a function, but making and evaluating any kinds of design are also undefined. Either there should be no tasks of making designs or its conventions must be urgently set up and taught.

- CS4. Former notations of functions might be applied both in the problems and in their solutions as well.

REMARK 1. Restrictions to notations must be treated carefully. It is a general rule that words, notations, etc. used in the text of a problem and in its solution must correspond to each other. Signs or notations which are difficult for the problem solver to understand or apply should not be used in the problem. E.g. letters in bold, or a borderline case is the use of arrows from bar.

REMARK 2. There are hardly any conventions for notations, although the increasing use of computers makes them more and more important. This is also supported by the following convention.

- CS5. We would need conventions, for example in the case of elementary functions (trigonometric, logarithmic), where bracketing arguments is quite problematic. A convention could be for instance that arguments that are not signs (but either letters or numbers) should be put in brackets.

- CS6. Words describing geometric concepts are often ambiguous, e.g. the word circle might be used to refer to a circle line or to a disc or the word segment might refer to the segment itself or its length. Even if there is a very little possibility of ambiguity a very accurate definition must be given. 
- CS7. Students must be familiar with the concept of locus (a set of points satisfying a particular criterion).

- CS8. When a die is thrown in probability problems it is equally likely to show any one of the six possible outcomes.

REMARK 1. Sometimes the text of a problem draws attention to the fact that the die is fair, other times it is not mentioned, which is very annoying. The problem solver does not know what to presume about the dice. The same problem exists when drawing balls of different colours.

REMARK 2. The fuzziest field in mathematics is the field of probability, but other fields must be clarified, too. E.g. statistics, graphs, analysis among new fields and solid geometry among former ones are fields requiring such a systematisation.

\subsection{Legal conventions}

- CL1. If a problem cannot be solved with literal interpretation of the text or applying conventions (e.g. using convention G2) (or it is so difficult that its solution cannot be expected), it must not put students at disadvantage.

REMARK. It also counts as a disadvantage if another student gets points for modifying and solving a problem.

REMARK. We admit that the statement CL1 might be debatable since there should not be such problems. Problems of this type may be given during the process of studying, but not in testing, or they may be included in tests only when students have been prepared well for them. Currently most teachers tell their students that all problems are solvable and the solution is always unambiguous, which, unfortunately, is not always true. 


\section{References}

[1] S. Kántor, Módszerek és elvárások (Methods and expectations), Stúdium Kiadó, Debrecen, 2001

[2] S. Kántor, Konvenciók (Conventions), A Matematika Tanítása, VII, no. 1-2 (1999), 35-37.

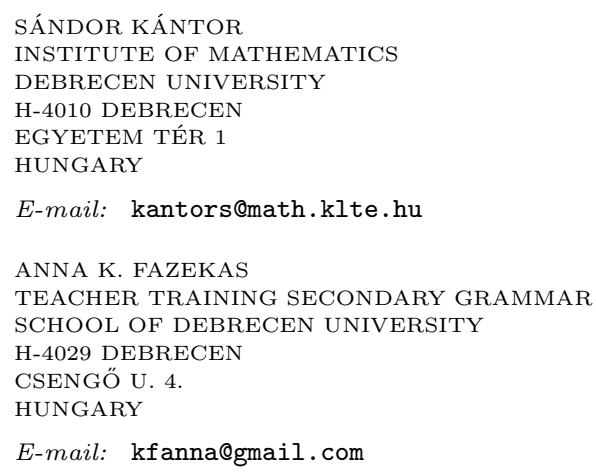

(Received December, 2010) 